\title{
Gravitational oscillations of a liquid column in a pipe
}

\author{
Élise Lorenceau and David Quéréa) \\ Laboratoire de Physique de la Matière Condensée, URA 792 du CNRS, Collège de France, \\ 75231 Paris Cedex 05, France \\ Jean-Yves Ollitrault \\ Service de Physique Théorique, CE Saclay, 91191 Gif-sur-Yvette Cedex, France \\ Christophe Clanet \\ Institut de Recherche sur les Phénomènes Hors Equilibre, UMR 6594 du CNRS, 49 rue F. Joliot-Curie, \\ BP 146, 13384 Marseille, France
}

(Received 29 June 2001; accepted 18 March 2002; published 3 May 2002)

\begin{abstract}
We report gravity oscillations of a liquid column partially immersed in a bath of liquid. We stress some peculiarities of this system, namely (i) the fact that the mass of this oscillator constantly changes with time; (ii) the singular character of the beginning of the rise, for which the mass of the oscillator is zero; (iii) the sources of dissipation in this system, which is found to be dominated at low viscosity by the entrance (or exit) effects, leading to a long-range damping of the oscillations. We conclude with some qualitative descriptions of a second-order phenomenon, which is the eruption of a jet at the beginning of the rise. (c) 2002 American Institute of Physics.
\end{abstract}

[DOI: $10.1063 / 1.1476670]$

\section{EXPERIMENT}

A vertical cylindrical glass pipe, closed at its top, is partially immersed in a large bath of liquid. The experiment consists of opening the pipe, and recording the height $Z$ of the liquid column as a function of time $T$ (Fig. 1). The pipe has a centimetric radius $R$ (which makes capillary effects negligible), and a total length of about $1 \mathrm{~m}$. We denote $H$ the depth of immersion, and $h$ the level of liquid inside the tube before opening. This parameter can be adjusted by adding with a syringe either liquid or air at the bottom of the column before opening the top. We are interested here in liquids of low viscosity $\eta$ (such as water or hexane), so that the motion of the liquid is dominated by inertia and gravity, leading to numerous oscillations of the liquid column.

Figure 2 shows typical observations of the column height as a function of time, obtained thanks to a high speed camera ( $\sim 125$ frames per second). For this particular experiment, the immersion depth was $H=30 \mathrm{~cm}$, the tube radius $R=1 \mathrm{~cm}$, and the initial height of liquid inside the column $h=3 \mathrm{~mm}$. The liquid was hexane, of density $\rho=660 \mathrm{~kg} / \mathrm{m}^{3}$ and viscosity $\eta=0.39 \mathrm{mPa}$. Both the height and the time are made dimensionless in Fig. 2, using the natural length and time scales, namely $H$ and $\sqrt{H / g}$, where $g$ is the acceleration of gravity. We denote $z$ and $t$ as these reduced quantities. We can observe in Fig. 2 several features, on which we shall base our discussions: (i) the height first quickly increases (the typical velocity at the beginning is $170 \mathrm{~cm} / \mathrm{s}$ ), and reaches a maximum $z_{M}=1.52 \pm 0.01$ for $t=3.0 \pm 0.5$; (ii) then, many oscillations are observed, before approaching the final equilibrium height $z=1$; the damping is not exponential, since the ratio between two successive maxima of the

${ }^{a)}$ Electronic mail: quere@ext.jussieu.fr function $(z-1)$ is not a constant (the four first ratios are, respectively, $0.61,0.68,0.73$, and 0.77 , and increase with time); (iii) a pseudoperiod can be deduced from the data, which is $6.3 \pm 0.5$; this period is quite well defined for the first oscillations, but slightly increases (of typically less than $5 \%)$ at longer times.

We shall first describe the principal characteristics of a model recently proposed to analyze the nonlinear oscillations of a liquid column. Then, we shall discuss different effects such as the speed of invasion, the initial acceleration of the fluid, and the damping. We shall conclude with qualitative observations related to local properties of the flow.

\section{MODEL}

A model was recently proposed to describe the capillary motion of a wetting liquid inside a small vertical tube initially empty $(H=h=0)$, in the inertial regime. ${ }^{1}$ Then, the forces acting on the liquid column write $2 \pi R \gamma-\rho g \pi R^{2} Z$ (denoting $\gamma$ as the liquid surface tension). Here, the tube radius is much larger than the (millimetric) capillary length, so that capillary forces can be neglected and replaced by the hydrostatic pressure as a driving force. Hence, the total force $F$ acting on the liquid column is found to exhibit a structure very similar to the one in the capillary problem:

$$
F=\rho g \pi R^{2}(H-Z) \text {. }
$$

It is very instructive to consider first a situation without any source of dissipation. Then, the total energy $E$ of the column is the sum of the kinetic energy and the potential energy $U$ which can be integrated from $F$ (taking $U=0$ for $Z=0)$. Hence it is expressed as

$$
E=\frac{1}{2} \rho \pi R^{2} Z \dot{Z}^{2}+\frac{1}{2} \rho g \pi R^{2} Z^{2}-\rho g H \pi R^{2} Z .
$$


(a)

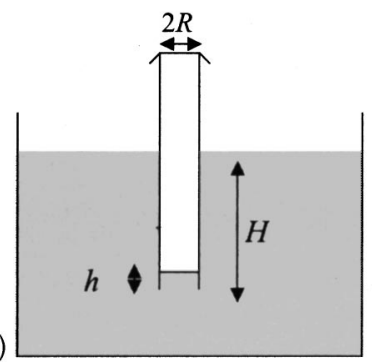

(b)

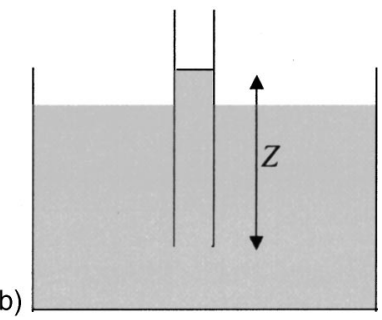

FIG. 1. Sketch of the experiment: (a) before opening the top $(T<0)$; (b) when the motion takes place $(T>0)$.

In dimensionless variables (and scaling the mass by $\rho \pi R^{2} H$ ), it reads

$$
e=\frac{1}{2} z \dot{z}^{2}+\frac{1}{2} z^{2}-z \text {. }
$$

Considering $e$ as a constant with time, Eq. (3) can be integrated, which leads to parabolic oscillations of the equation: $z(t)=\sqrt{2} t(1-t / 4 \sqrt{2})$, supposing $z(0)=0$. The maximum, reached at $t=2 \sqrt{2}$, is $z=2$, far above the maximum observed in the experiment in Fig. 2. Assuming energy conservation makes this parabolic behavior periodic (with a peculiarity: when $z$ comes back to zero, the velocity is maximum but the mass is zero: there is no inertia and the liquid column can bounce)_but observations clearly reveal a damping of the oscillations.

The second step consists of analyzing the possible causes of dissipation in the system. We could try to incorporate the viscous dissipation along the wall of the tube, but this should be negligible at short time, i.e., at a time scale smaller than $\rho R^{2} / \eta$, the characteristic time for setting a Poiseuille profile in the tube. This time in these experiments is very large, typically $10^{2}-10^{3}$ in our dimensionless units. The negligible influence of viscosity at short time was confirmed by doing the same experiment with water (three times as viscous as hexane), for which we found exactly the same positions for the five first maxima and five first minima (within $1 \%$ in error).

In classical textbooks, ${ }^{2}$ one can find that a second cause of dissipation for a liquid of very small viscosity is the sin-

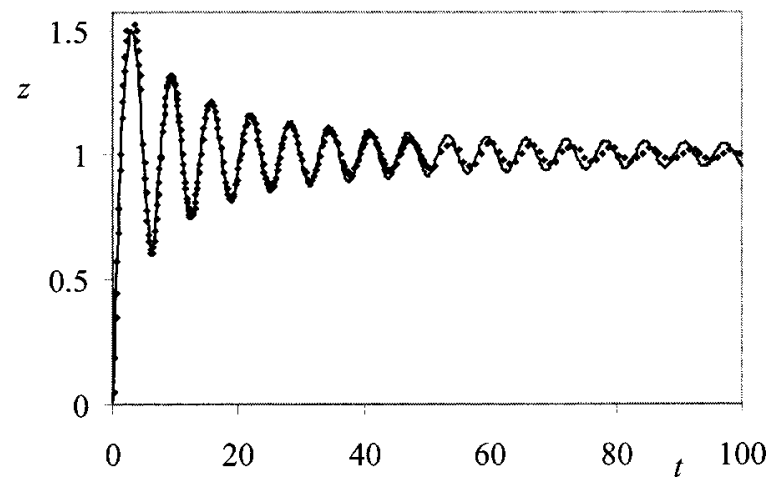

FIG. 2. Height of the liquid column vs time, for a glass tube $(R=1 \mathrm{~cm})$ partially immersed at a depth $H=30 \mathrm{~cm}$ in hexane. Initially $(t=0)$, the tube is empty. The height is normalized by $H$, and the time by $(H / g)^{1 / 2}$. The dots correspond to experimental data and the full line to a numerical integration of Eq. (9). gular pressure loss at the tube entrance (if the liquid rises) or exit (if the liquid goes down). This pressure loss is due to the difference of radii between the tank (of huge radius) and the tube (of much smaller radius): because of the abrupt contraction between both, some eddies appear at the entrance of the tube, dissipating a certain amount of energy. This pressure loss is classically evaluated by applying the Bernoulli equation (based on the conservation of energy) and the Euler equation (based on the momentum equation) to the liquid column, which does not lead to the same result. ${ }^{2}$ The difference between these results is the pressure loss. When the ratio of the tube area to the tank area is close to zero (in our experiment, this ratio is of the order of $10^{-2}$ ), the singular pressure loss $\Delta P$ has a very simple expression: ${ }^{2}$

$$
\Delta P=\frac{1}{2} \rho \dot{Z}^{2} .
$$

This pressure loss is positive and is simply equal to the kinetics energy per unit volume of the column. The associated energy loss is negative, and has a different sign depending on whether the liquid is going up $(d Z>0)$ or down $(d Z<0)$. In dimensionless quantities, the energy loss is thus expressed as

$$
d e=\frac{1}{2} \dot{z}^{2} d z
$$

when the liquid falls $(d z<0)$, and

$$
d e=-\frac{1}{2} \dot{z}^{2} d z
$$

when it rises $(d z>0)$. If the situation is quite clear at the descent [Eq. (5a) just expresses the loss of kinetic energy associated with the loss of a fluid jet entering an infinite pool of the same fluid], it is not the case for the rise, and it has been proposed to introduce a numerical empirical coefficient $K$ for the energy loss in this case:

$$
d e=-\frac{1}{2} K \dot{z}^{2} d z,
$$

where $K$ should be in the interval $[0,1]$. We shall see that our experiments are well described by taking $K=1$, but we shall discuss how the results should be modified for smaller values of this coefficient.

We first consider the case where the singular pressure loss at the tube entrance is the main cause of dissipation and thus neglect the viscous dissipation along the pipe wall. Differentiating Eq. (3) with respect to $t$, and equating the resulting expression with either Eq. (5a) or (5b), we find two different equations, depending on the direction of the motion:

$$
\begin{aligned}
& z \ddot{z}+\dot{z}^{2}=1-z \text { for } d z>0, \\
& z \ddot{z}=1-z \text { for } d z<0 .
\end{aligned}
$$

It is worth noting that Eq. (6a) just expresses Newton's law of dynamics, for a system of mass $M$ and velocity $V$ driven by a force $F$ :

$$
\frac{d}{d t} M V=F-M g .
$$

For the descent, a similar law can be written, taking into account the thrust associated with the emission of a jet coming out of the pipe at a velocity $V$. Thus, Eq. (7a) must be corrected in 


$$
\frac{d}{d t} M V=F-M g+\dot{M} V
$$

which is just Eq. (6b), with dimensions. Note that we did not consider a term of the form $\dot{M} V$ in Eq. (6a) because the mass radiates from all the directions in the reservoir to enter the tube, while the jet is directional at the exit: the thrust at the entrance implies a nearly zero velocity, and thus is itself nearly zero. This defines the case $K=1$ stated in Eqs. (5b) and $(5 \mathrm{c})$ - and this result should depend on the shape of the pipe. For example, $K$ should decrease for funnel-shaped pipes, which would drive more smoothly the current lines. Note also that Bernoulli, considering in his book on Hydraulics $^{3}$ the question of a pipe emptying in a bath, proposed Eq. (6b) by writing directly Newton's law with the form: $M d V / d t=F-M g-$ a straightforward derivation, indeed, but quite hazardous since $M$ is changing with time.

The energy loss associated with Eqs. (7a) and (7b) can be calculated in a general way. The energy $E$ is $1 / 2 M V^{2}$ $+U$, denoting $U$ as the potential from which the forces can be derived. Using Eq. (7), the way the energy varies as a function of time can be deduced, and a unique expression is found for both the rise $(\dot{M}>0)$ and the descent $(\dot{M}<0)$ :

$$
\frac{d E}{d t}=-\frac{1}{2}|\dot{M}| V^{2}
$$

Equation (8) is found to be identical to Eqs. (5a) and (5b). It expresses more generally the energy loss related with an entrained mass $(d E / d t=0$ if $\dot{M}=0)$. It thus concerns similar questions such as the bursting of a soap film ${ }^{4}$ or even the academic problem of a rope wound on a pulley and drawn by a constant weight.

Equations (6a) and (6b) can eventually be integrated once, introducing two constants $A$ and $B$ :

$$
\begin{aligned}
& \frac{1}{2} z^{2} \dot{z}^{2}+\frac{1}{3} z^{3}-\frac{1}{2} z^{2}=A, \\
& \frac{1}{2} \dot{z}^{2}+z-\ln z=B .
\end{aligned}
$$

If $z=0$ at $t=0$, the constant $A$ is zero, and Eq. (9a) can be integrated once again, which provides the trajectory of the liquid column:

$$
z(t)=t\left(1-\frac{t}{6}\right)
$$

Thus, the beginning of the rise should be linear $(z \sim t$, for $t$ $\ll 6$ ), before the weight makes the velocity smaller and the trajectory parabolic. The maximum is reached for $t=3$, and is found to be $z_{M}=1.5$. The latter point is in close agreement with the data displayed in Fig. 2, which stresses that indeed energy loss is present in the system, even at short time.

Note that if we take as an expression for the energy loss at the rise the more general expression $(5 \mathrm{c})$, the maximum height can also be calculated analytically. We find: $z_{M}=(K$ $+2) /(K+1)$, which varies between 2 and $3 / 2$ when $K$ varies between 0 and 1 . The first value corresponds to energy conservation [Eq. (3) and below], while the second to the maximum of singular pressure loss. Our experimental data exhibit

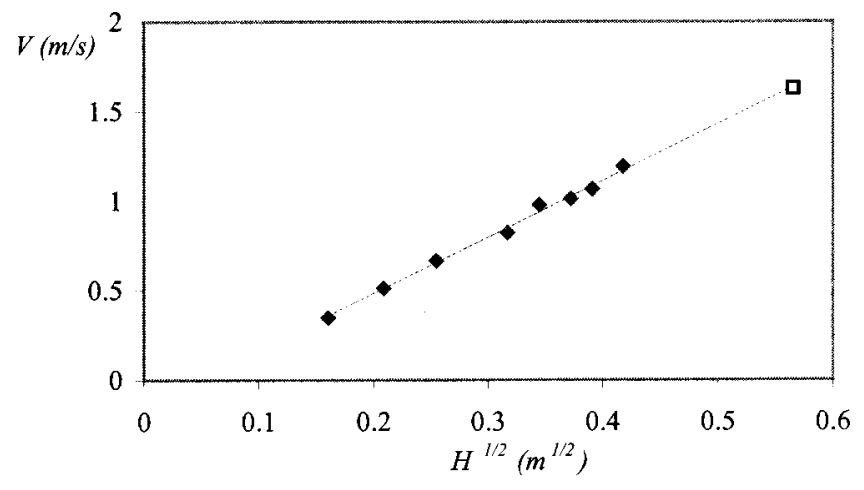

FIG. 3. Rise velocity $V$ of the liquid column at short time $(t<1.5)$, as a function of the square root of $H$, the depth of immersion in water (closed diamonds) or in hexane (open square). The full line has a slope $\sqrt{g}$, as expected from Eq. (11).

a maximum very close to 1.5 (but slightly larger, as expected since $K=1$ is the maximum possible value), so that we shall take $K=1$ in all the rest of this study.

We now focus on different peculiarities of this system in order to discuss more carefully some details of the model.

\section{DISCUSSION}

\section{A. Constant velocity regime}

Initially, the beginning of the rise is linear, which can be explained by balancing inertia with the pressure force $\left(\rho g H \pi R^{2}\right.$ ) exerted on the liquid column. This behavior is reminiscent of similar systems with a mass varying linearly with $z$, and driven by a constant force and resisting inertially. This indeed leads to a constant velocity, as observed for the retraction of a liquid sheet, ${ }^{5}$ the bursting of a soap film, ${ }^{4,6}$ the dewetting of a film of small viscosity ${ }^{7}$ and the first steps of capillary rise. ${ }^{8,9}$ Note that in all these problems, conservation of energy also leads to a constant velocity, but similarly overestimates the numerical coefficient of this velocity. ${ }^{10}$

We measured the initial velocity of the liquid column as a function of the depth of immersion $H$. Since the dimensionless law at short time $(t \ll 6)$ just reads $z=t$, introducing dimensional quantities implies a quick variation of the column velocity with $H$. Then, Eq. (10) just is expressed as

$$
Z(T)=\sqrt{g H} T .
$$

We did experiments with hexane, and found that indeed the height $Z$ of the liquid column increases linearly with time at short time (practically for $t<1.5$, which corresponds to 10 data points). Thus, we could report its velocity $V$ as a function of the square root of the depth height (Fig. 3), varying $H$ from 2 to $35 \mathrm{~cm}$, and indeed found a linear relation with a slope $\sqrt{g}$, as predicted by Eq. (11). Conservation of energy in Eq. (3) for a system starting from $z=0$ also predicts a regime of constant velocity, but with a higher slope $(\sqrt{2 g}$ instead of $\sqrt{g}$ ). Thus this regime of constant velocity also allows us to stress the existence of an energy loss in this system. Note also that the observed curve does not intercept the origin, which will be shown to be due to an entrance effect, characterized by a length of order $R$. Thus, our model only holds in the limit $H \gg R$. 


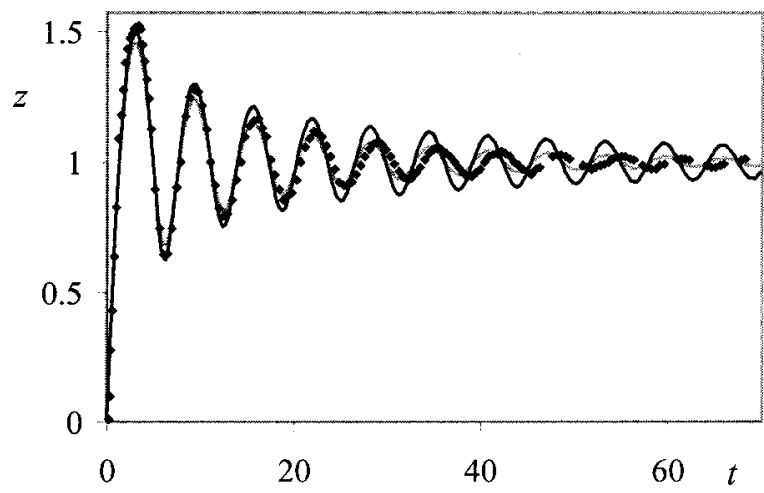

FIG. 4. Same experiment as in Fig. 2, in a thinner tube $(R=5 \mathrm{~mm})$. The dots are experimental data obtained with hexane. The full line corresponds to a numerical integration of Eq. (9), and the thin one to an integration of Eq. (17).

More generally, Eqs. (6a) and (6b) indicate that a solution of constant velocity can only be found at the rise and if gravity can be neglected $(d z>0$ and $z \ll 1)$, or in the case where a horizontal pipe is connected with the bottom of a very large tank, which only generates an entrance flow. On the other hand, Eq. (6b) shows that the velocity is never constant during the descent, and the only analytical regime in this case is a regime of constant acceleration: leaving a liquid column flow downwards from a very large height $\left(z_{0} \gg 1\right)$ yields $\ddot{z}=-1$.

\section{B. Oscillations, and their two regimes of damping}

At longer times, gravity cannot be neglected and Eqs. (9a) and (9b) can be integrated numerically. This solution is drawn in full line in Fig. 2, and compared with data obtained with hexane (for $H=30 \mathrm{~cm}$ and $R=1 \mathrm{~cm}$ ).

The agreement between the theory and the experiment is excellent during the first oscillations: both the positions of the extrema and the periodicity are well predicted by the model. In particular, the first half-oscillation is the parabola derived in Eq. (10). After typically ten oscillations, a slight shift appears, and the damping is observed to be quicker than predicted. We interpret this "overdamping" as due to the usual viscous friction along the tube, which must be taken into account as soon as a parabolic Poiseuille-Hagen profile has been established. This is achieved after the time necessary for the boundary layer to diffuse on a length $R$, which scales as $\rho R^{2} / \eta$, with a numerical coefficient of order 0.11 , as shown in Ref. 11. This time mainly depends on the tube radius, which can be easily checked by doing the same experiment in a thinner tube. Figure 4 shows the data obtained using a tube twice thinner $(R=5 \mathrm{~mm})$.

While the first oscillations remain quite well described by Eq. (9), it is indeed observed that the overdamping takes place much earlier: deviations toward Eq. (9) are observed around $t=15$, instead of $t=60$ (in agreement with the scaling for the time of diffusion of the viscous boundary layer, in $R^{2}$ ).

One of the remarkable features of this system is the persistence of the oscillations (typically, more than 20 oscillations can be observed). This is due to the particular source of

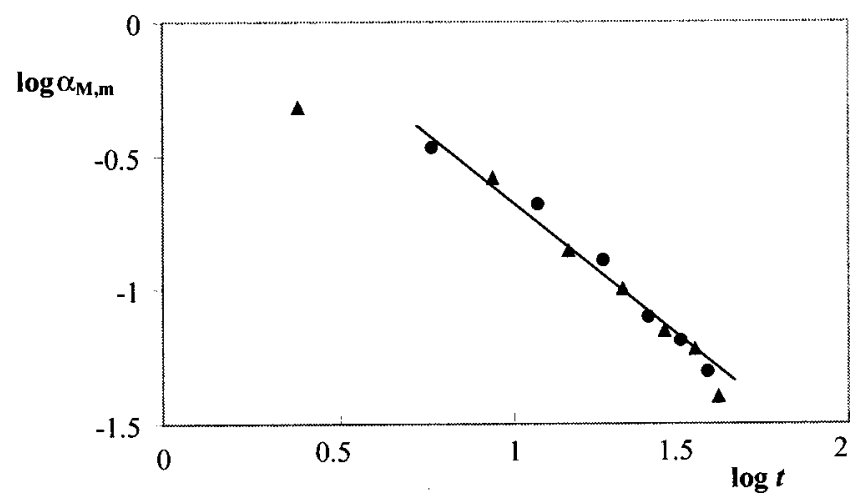

FIG. 5. Successive maxima $\alpha_{M}$ (triangles) and minima $\alpha_{m}$ (circles) of the oscillation amplitude as a function of time. The data are taken from Fig. 2 and the full line has a slope -1 .

dissipation in Eq. (9). If the damping were just caused by the viscous dissipation along the pipe, this would provide a decreasing exponential law for the maxima. In the case we are mainly interested in (short time behavior), the damping is due to the singular pressure loss at the entrance (or exit) of the pipe. The following argument allows us to understand why it is so low. From Eqs. (3) and (5), we can derive an equation for the energy loss:

$$
\frac{d}{d t}\left(z \dot{z}^{2}+(z-1)^{2}\right)=-|\dot{z}| \dot{z}^{2} .
$$

We set $z(t)=1+\alpha(t) \sin t$, with $\alpha \ll 1$, and suppose a slow variation for $\alpha$. During a period, the mean value of the quantities $|\dot{z}| \dot{z}^{2}$ and $\left(z \dot{z}^{2}+(z-1)^{2}\right)$ are $4\left|\alpha^{3}\right| / 3 \pi$ and $\alpha^{2}$, respectively. Thus, an equation for the oscillation amplitude $\alpha$ is obtained from Eq. (12):

$$
\frac{d \alpha^{2}}{d t}=-\frac{4\left|\alpha^{3}\right|}{3 \pi}
$$

which yields

$$
\alpha(t)= \pm \frac{3 \pi}{2 t} .
$$

Even if this linear approximation should mainly concern the oscillations of small amplitude, it helps to understand that the damping is unusually long, due to this hyperbolic behavior. Furthermore, a hyperbolic damping is in fair agreement with our data even for oscillations of non-negligible amplitude, as shown in Fig. 5 where the maxima and minima corresponding to Fig. 2 are displayed versus time in a log$\log$ plot.

It is observed that (apart from the first maximum), the damping is close to being hyperbolic in time (the full line indicates the slope -1 ), before accelerating (the two last points), because of the additional dissipation due to the liquid viscosity. The latter can of course be evaluated by incorporating in the model a viscous Poiseuille friction along the pipe. If the liquid front progresses by a length $d z$, the corresponding energy loss writes (in the same dimensionless variables as previously)

$$
d e=-\Omega z \dot{z} d z,
$$


where the number $\Omega$ compares viscosity with inertia:

$$
\Omega=\frac{16 \eta H^{1 / 2}}{\rho R^{2} g^{1 / 2}} .
$$

A difference with the energy loss due to pressure entrance is that de (energy variation associated with a motion $d z$ of the column) has the same expression whatever the direction of the motion, since $\dot{z}$ and $d z$ always have the same sign. Taking into account this viscous friction modifies Eq. (6), which becomes

$$
\begin{aligned}
& z \ddot{z}+\dot{z}^{2}=1-z-\Omega z \dot{z} \quad \text { for } d z>0, \\
& z \ddot{z}=1-z-\Omega z \dot{z} \quad \text { for } d z<0 .
\end{aligned}
$$

Unlike Eq. (6), Eq. (17) cannot be integrated analytically, but only numerically: such an integration is performed in Fig. 4 (in thin line). The resulting curve fits quite well the extrema of the oscillations, but a shift in time appears, which remains unexplained. The use of a simple Poiseuille friction law for this oscillatory behavior could be questioned. The dissipation in the menisci could also become non-negligible in these regimes of approach of the equilibrium.

In the particular case of very large $\Omega$, inertia can be neglected, and the equation for the column motion simply is expressed as

$$
\Omega_{z} \dot{z}=1-z
$$

which is often referred to, in the context of dynamic capillary rise, as the Washburn equation. ${ }^{12}$ At short time (but large enough so that inertia can be neglected), $z$ is small $(z \ll 1)$, and integration of Eq. (18) shows that the rise follows a diffusion-type law: $z(t)=\sqrt{t / \Omega}$. Then, when approaching equilibrium $(z \rightarrow 1)$, we find an exponential relaxation: $z(t)$ $=1-\exp (-t / \Omega)$.

An interesting feature of Eq. (17) is that it allows us to predict if the system will exhibit oscillations, or not. We saw that at large viscosities $(\Omega \gg 1)$, the system just relaxes toward equilibrium, without any overshoot of the equilibrium height. Thus, a critical number $\Omega_{c}$ does exist, below which oscillations develop. Close to $\Omega_{c}$, we can linearize Eqs. (17a) and (17b), which both reduce to

$$
\ddot{\zeta}+\Omega \dot{\zeta}+\zeta=0
$$

where we have set: $z=1+\zeta$, with $\zeta \ll 1$. This equation only leads to oscillations if $\Omega<\Omega_{c}=2$, i.e., for small enough viscosities. Written dimensionally on the depth of immersion, this criterion reads

$$
H<H_{c}=\frac{\rho^{2} g R^{4}}{64 \eta^{2}} .
$$

This criterion is largely fulfilled in the series of experiments presented previously: with water and centimetric tubes, $H_{c}$ is of order $1 \mathrm{~km}$ ! But this height rapidly decreases when making the tube thinner: for a tube of radius $3 \mathrm{~mm}$ and a liquid 10 times more viscous than water, $H_{c}$ becomes of order $10 \mathrm{~cm}$.

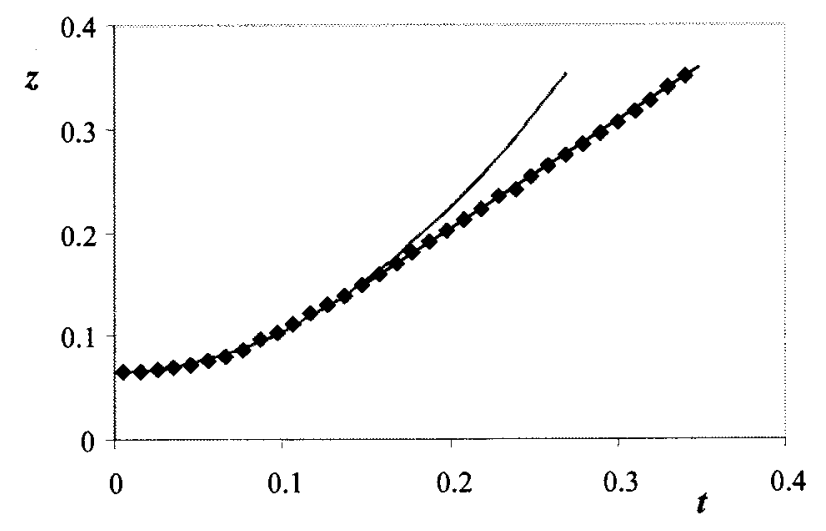

FIG. 6. Height $z$ vs time $t$, in the very first steps of the rise $(R=20 \mathrm{~mm}$, $H=30 \mathrm{~cm}$, and $h=1.9 \mathrm{~cm}$ ). The data, obtained with water, are successively fitted by a parabola of equation $z(t)=a+t^{2} / 2\left(a+z_{0}\right)$ [Eq. (24)], from which the coefficient $z_{0}$ can be deduced, and by a straight line of equation $z(t)=t$ [Eq. (11)].

\section{Very short time behavior}

\section{Starting of the liquid column}

Let us come back to the beginning of the rise, starting from $z=0$. We showed that it obeys a very simple law, since the height of the column increases linearly with time [Eq. (11) and Fig. 2]. An interesting question is the way the system finds its constant velocity $V$. At $t=0$, the system is at rest and there is a regime of transition during which the velocity quickly increases from 0 to $V$. Then, the column weight is negligible, and Eq. (6a) can be written as

$$
z \ddot{z}+\dot{z}^{2}=1 \text {. }
$$

This equation has no solution which verifies both $z=0$ and $\dot{z}=0$ for $t=0$, because of the singularity at $z=0$ [then, a zero velocity implies an infinite acceleration for Eq. (21) to be obeyed]. But physically, this singularity does not exist, because of the mass of liquid entrained below the pipe. Thus, Eq. (21) can be rewritten, taking into account this additional mass from the beginning:

$$
\left(z+z_{0}\right) \ddot{z}+\dot{z}^{2}=1 \text {, }
$$

where $z_{0}$ is the height below the pipe where the liquid is entrained. Because the velocity field in the bath quickly vanishes as a function of the distance to the entrance, we expect $Z_{0}$ (the dimensional version of $z_{0}$ ) to be of order $R$, the radius of the tube. More precisely, by integrating the velocity profile from the entrance of the tube to infinity, Szekeley et al. (in the context of capillarity) ${ }^{13}$ calculated an entrance length $Z_{0}=7 / 6 R$.

The general solution of Eq. (22) can be written as

$$
z(t)+z_{0}=\sqrt{\left(a+z_{0}\right)^{2}+t^{2}}
$$

denoting $a=h / H$ as the height of liquid initially present in the tube [as sketched in Fig. 1(a)]. At very short time, Eq. (23) leads to a parabolic behavior (acceleration stage):

$$
z(t) \approx a+\frac{t^{2}}{2\left(a+z_{0}\right)} .
$$




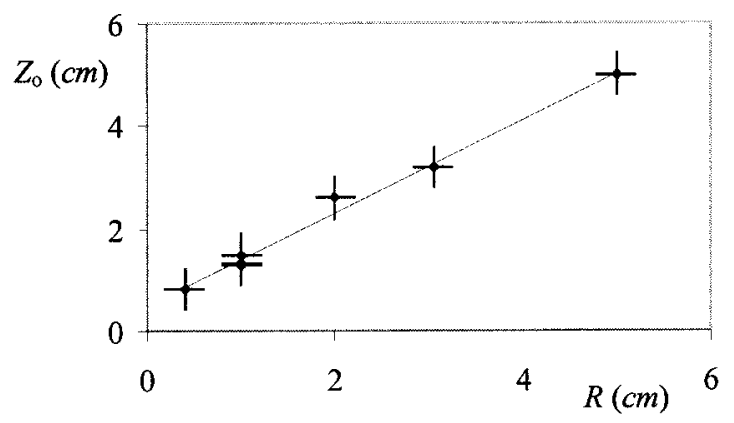

FIG. 7. Entrance length $Z_{0}$ vs $R$, the radius of the tube $(H=20 \mathrm{~cm}$ and $h$ $\approx 1 \mathrm{~cm}$ ). The data are obtained with water.

Later $\left(t \gg a+z_{0}\right)$, it matches the solution of constant velocity $z=t$ analyzed previously (Fig. 3).

By taking pictures at a high rate (typically 1000 frames per second), we could record the very beginning of the rise. Such data are reported in Fig. 6. It is observed that the behavior at a very short time $(t<0.15)$ can indeed be fitted by a parabola [Eq. (24)], from which two coefficients can be deduced. One ( $a=0.064$, in Fig. 6) is indeed found to be the initial height of liquid in the tube, while the second $\left(a+z_{0}\right.$ $=0.126$, in Fig. 6) provides the value of $z_{0}$. Note that at larger time, the parabolic regime meets the linear one discussed in Sec. III A [Eq. (11)].

We plotted in Fig. 7 the value of $Z_{0}$ (deduced from fits such as the one in Fig. 6), as a function of $R$, the pipe radius. The results are found to agree closely with Szekeley's predictions: ${ }^{13} Z_{0}$ varies linearly with $R$, with a numerical coefficient of order 1.

We also considered the influence of $h$ on $Z_{0}$, and focused on the case of an empty tube $(h \rightarrow 0)$. Then, as stressed previously, the problem should become singular. Practically, it is not; Fig. 8 shows that $Z_{0}$ does not depend on $h$, which is consistent with the hypothesis of an added mass below the tube entrance. Even in the limit of a tube initially empty $(h \rightarrow 0)$, the mass of accelerated fluid is not zero and the acceleration remains finite.

Since the flow inside the tube perturbs the reservoir on a length of order $R$, all the conclusions and interpretations presented previously (Secs. III A and III B), for which we had

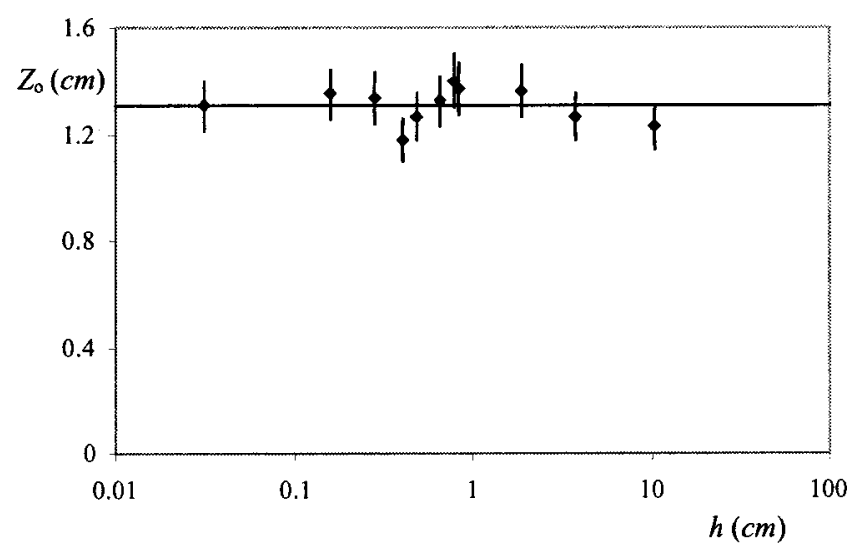

FIG. 8. Entrance length $Z_{0}$ vs $h$ the height of water initially present at the bottom of the pipe $(R=1.2 \mathrm{~cm}$ and $H=30 \mathrm{~cm})$.

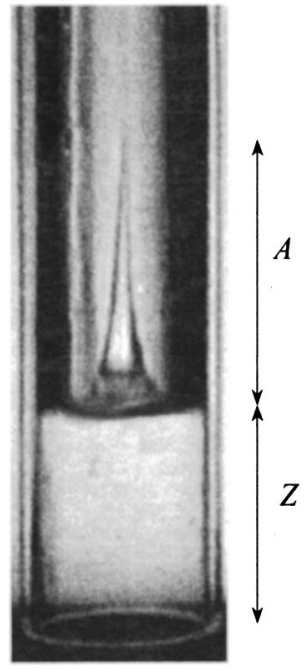

FIG. 9. Early stage of the rise ( $R=20 \mathrm{~mm}, H=30 \mathrm{~cm}$, and $h=0 \mathrm{~mm})$. The front is flat, except at the tube center where a liquid (here, water) finger develops.

$Z \gg R$, remain unchanged. The corrections mainly concern the very first steps of the trajectory, in the accelerating regime illustrated in Fig. 6. Other modifications are quite negligible: for example, the position $z_{M}$ of the first maximum is found to be slightly modified by taking into account the entrance length, from 1.5 to $1.5+z_{0} / 2$ (i.e., about 1.53 for the data in Fig. 2, very close to the observed value).

\section{Jet eruption}

We have up to now focused our discussion on the motion of the whole column, but local deformations of the free surface were also observed at short time. Figure 9 shows a side view of the tube for $Z$ of about $2 R$, where it can be seen that a liquid finger develops at the center of the tube. This finger rises during the first oscillation and collapses before $z$ reaches its maximum value $z_{M}$; this structure is local and does not impact the more macroscopic observations reported earlier.

The maximum size $A$ of the finger depends on the height $h$ of liquid initially in the tube, as shown in Fig. 10. Note that

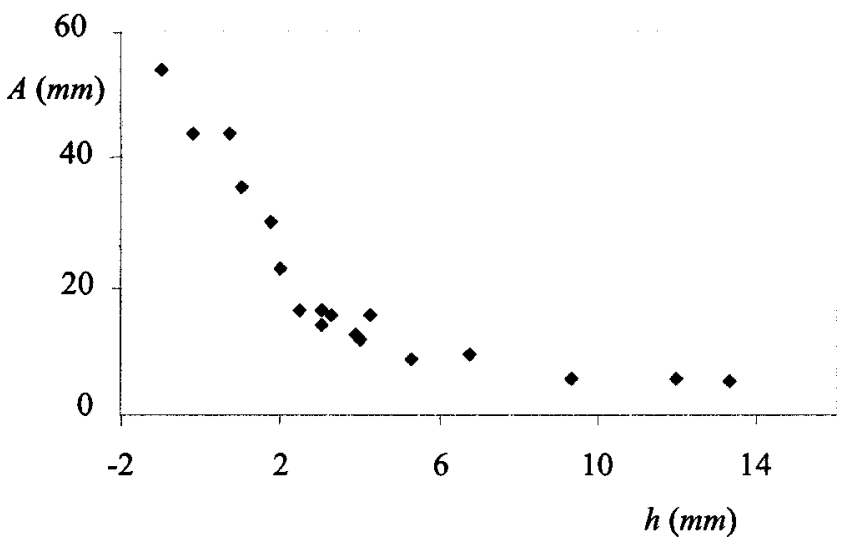

FIG. 10. Maximum amplitude of the water finger vs the initial height of liquid $h$. The experiment was carried out in a tube of radius $R=20 \mathrm{~mm}$ and for $H=30 \mathrm{~cm}$. 


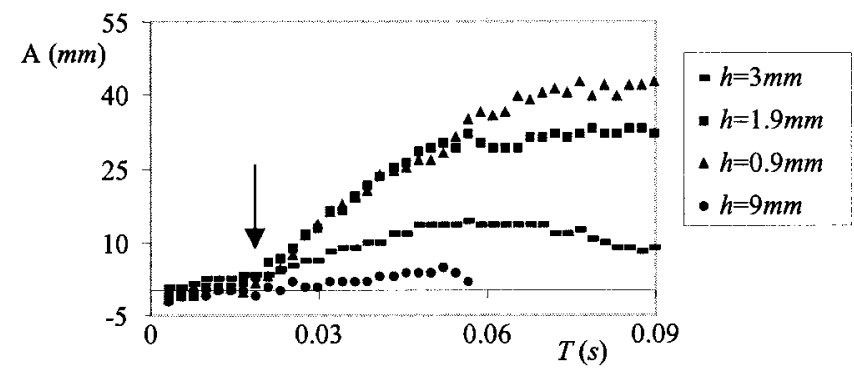

FIG. 11. Amplitude of the water finger as a function of time $(H=30 \mathrm{~cm}$ and $R=20 \mathrm{~mm}$ ). The liquid column starts rising at $T=0$ and the finger starts developing at the arrow.

$h$ can even been made negative, by injecting air bubbles inside the empty pipe, before the rise takes place.

For large $h(>6 \mathrm{~mm})$, the size of the liquid finger does not depend on $h$. In this regime, we still observe some oscillations of the interface due to the abrupt contraction between the reservoir and the tube. Such oscillations were described by Taylor, ${ }^{14}$ in the case of a tank with an oscillating wall. $\mathrm{He}$ showed that free standing waves could set up in the tank, with a shape very close to the one observed in the tube. For smaller $h$, a strong dependence can be observed: the smaller the height, the longer the finger. We were interested in the dynamics of the finger growth. Figure 11 reports different series of experiments.

Besides the $h$ dependence of $A$ stressed previously, Fig. 11 shows that the finger grows after some delay (typically $0.02 \mathrm{~s}$ ), whatever $h$. This implies that a simple scenario (either a convergence of the flow lines, or a kind of RayleighTaylor instability due to the pulse of acceleration at the beginning of the rise) cannot explain the phenomenon. To go further, we took detailed films of the very beginning of the rise, focusing on the shape of the front interface. A series of snapshots taken at short time from above the tube is displayed in Fig. 12.

These pictures show the existence of a circular rim, which sets near the wall of the tube, and closes as time goes on. The collapse of this surface wave produces a jet (last picture of the series), as observed in similar situations. ${ }^{15}$ The speed at which the liquid crater closes could be deduced from series similar to Fig. 12. Figure 13 shows how the diameter $D$ of the liquid crater varies versus time.

The liquid crater closes at a constant velocity, which is $1.3 \mathrm{~m} / \mathrm{s}$ in the above-given example, of the order of the velocity $\sqrt{g H}$ of the rising column $(1.4 \mathrm{~m} / \mathrm{s}$, in the same experiment). Note that this wave starts propagating during the acceleration phase of the column. We saw that in this phase, the acceleration is of the order of $g H / R$ (for $h=0$ ), signifi-

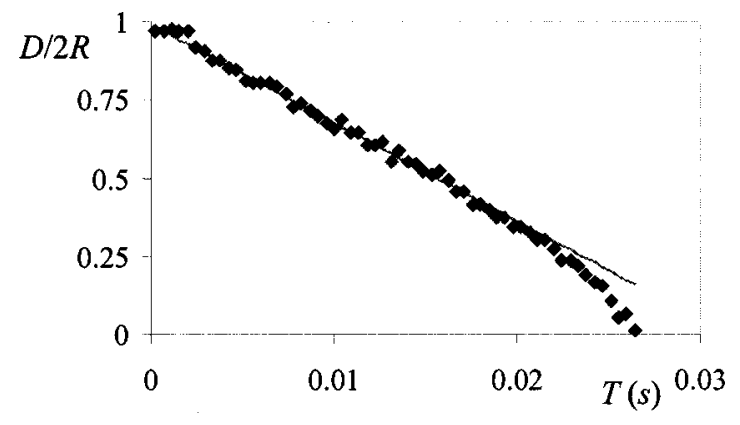

FIG. 13. Diameter of the water crater vs time $(R=20 \mathrm{~mm}$ and $H=20 \mathrm{~cm})$.

cantly larger than $g$. Hence, for a wave vector $k$, a typical wave velocity should scale as $\sqrt{g H / k R}$, of the order of $\sqrt{g H}$ for $k \sim 1 / R$. The time needed for shutting down the crater in a pipe of radius $20 \mathrm{~mm}$ is $26 \mathrm{~ms}$, of the order of the delay measured in Fig. 11.

The origin of the crater can also be questioned, and related to local flows at the pipe entrance. An effervescent drug placed below the tube provides indications on the flow: the gas bubbles reveal the existence of a circular vortex which remains close to the entrance as fluid sinks into the tube (Fig. 14). This vortex is related to the contraction of the flow lines entering the tube jet (the so-called vena contracta phenomenon), which traps some fluid at this place.

If $h$ is negative, the initial conditions are different. Then, some air can be trapped in the tube creating a vortex ring of air rather than a liquid one (as seen in the preceding paragraph). This phenomenon could be enhanced by placing a diaphragm at the tube entrance, as seen in Fig. 15. The column then adopts the diameter of the diaphragm, with a modulation of frequency $184 \mathrm{~Hz}$. (In addition, the finger previously described is still here, above the main column, as observed in Fig. 15.) The column modulation is probably due to the stationary pressure waves of the air trapped into the tube. The frequency of such a resonant tube (open at one end and closed at the other) is

$$
f_{n}=n C / 4 Y \text {, }
$$

where $C$ is the sound speed, $Y$ the total length of the tube (1.6 $\mathrm{m}$ in the experiment), and $n$ the mode of oscillation. For $n$ $=1$, we find $f_{1}=187 \mathrm{~Hz}$, in very good agreement with the measured frequency. This agreement remains excellent if the tube length is changed. Another cause of modulation of this cylindrical column of liquid could be the liquid surface tension (Plateau-Rayleigh instability), but it would lead to a
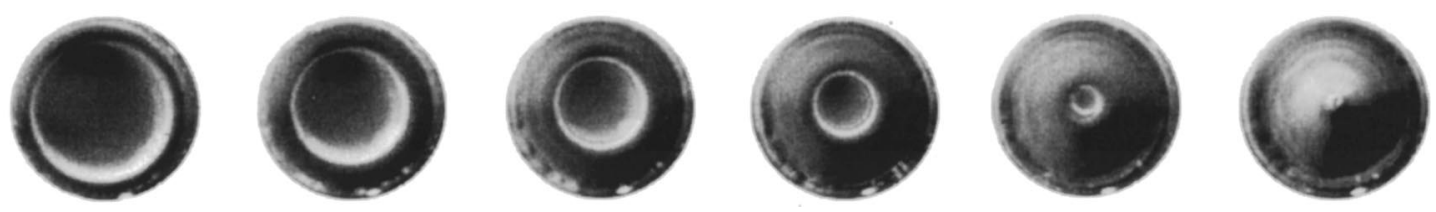

FIG. 12. Set of pictures taken from above each $2.7 \mathrm{~ms}(R=20 \mathrm{~mm}, H=20 \mathrm{~cm}$, and $h=3 \mathrm{~mm})$. These pictures correspond to the first points (before the arrow) in Fig. 11. It can be observed that a cavity forms and closes, producing a water jet (last picture). 

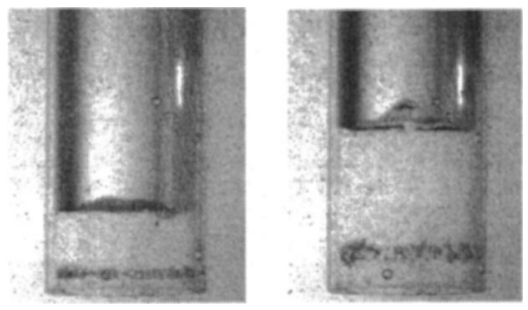

FIG. 14. Visualization of eddies using small bubbles as a tracer for the flow of water $(R=20 \mathrm{~mm}$ and $h=7 \mathrm{~mm})$. The pictures are taken before the eruption of the jet.

totally different wavelength (higher than the column perimeter, i.e., of the order of $10 \mathrm{~cm}$ instead of the observed centimeter in Fig. 15).

\section{CONCLUSION}

We have studied the gravitational oscillations of a liquid column initially empty (or nearly empty) and partially immersed inside a large reservoir. We have stressed that this problem has different analytical solutions, depending on the liquid viscosity. For very viscous liquids, the rise should obey the classical laws of impregnation (height proportional to the square root of time, followed by an exponential relaxation toward equilibrium). But the interesting case is the low viscosity limit, for which different features were observed and analyzed, focusing in particular on the first steps of the rise (inertial regimes): after an accelerating phase (where the

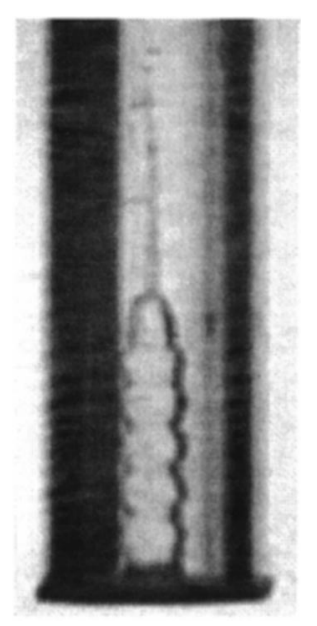

FIG. 15. Water column rising in a tube $(R=20 \mathrm{~mm})$ where a diaphragm (of radius $10 \mathrm{~mm}$ ) partially closes the bottom. liquid entrained was mainly the one below the tube), the velocity of rise was found to be a constant fixed by the depth of immersion. Then, the rise was observed to slow down (because of the column weight); we have shown that the trajectory is parabolic, reaching as a first maximum 1.5 times the depth of immersion. This value confirms that energy is indeed dissipated in this inertial phase, because of the sudden contraction endured by the moving liquid which passes from a large reservoir to a finite pipe. After this first maximum, many rebounds were observed, which was understood by evaluating the long range damping associated with this energy loss: the envelope of the height/time dependence was found to be hyperbolic (instead of exponential, as it is the case for usual viscous damping). At long time, viscosity must of course also be considered, which provides a quicker damping of the oscillations. We finally described qualitatively an instability of the liquid/air interface during the first steps of the rise: then, a liquid jet is emitted while the column develops. A complete study of this jet remains to be done.

\section{ACKNOWLEDGMENTS}

We thank Marc Rabaud, Élie Raphael, Konstantin Kornev, and Alexander Neimark for very useful discussions.

${ }^{1}$ D. Quéré, É. Raphaël, and J. Y. Ollitrault, "Rebounds in a capillary tube," Langmuir 15, 3679 (1999).

${ }^{2}$ G. K. Batchelor, An Introduction to Fluid Dynamics (Cambridge University Press, Cambridge, 1967).

${ }^{3}$ D. Bernoulli, Hydraulics (Dover, New York, 1968).

${ }^{4}$ A. B. Pandit and J. F. Davidson, "Hydrodynamics of the rupture of thin liquid films," J. Fluid Mech. 212, 11 (1990).

${ }^{5}$ G. I. Taylor, "The dynamics of thin sheets of fluid," Proc. R. Soc. London, Ser. A 253, 313 (1959).

${ }^{6}$ F. E. C. Culick, "Comments on a ruptured soap film," J. Appl. Phys. 31, 1128 (1960).

${ }^{7}$ A. Buguin, L. Vovelle, and F. Brochard-Wyart, "Shocks in inertial dewetting," Phys. Rev. Lett. 83, 1183 (1999).

${ }^{8}$ D. Quéré, "Inertial capillarity," Europhys. Lett. 39, 533 (1997).

${ }^{9}$ K. G. Kornev and A. V. Neimark, "Spontaneous penetration of liquids into capillaries and porous membranes revisited," J. Colloid Interface Sci. 235, 101 (2001).

${ }^{10}$ P. G. de Gennes, "Mechanics of soft interfaces," Faraday Discuss. 104, 1 (1996).

${ }^{11}$ H. Schlichting, Boundary Layer Theory (McGraw-Hill, New York, 1968).

${ }^{12}$ E. W. Washburn, "The dynamics of capillary flow," Phys. Rev. 17, 273 (1921).

${ }^{13}$ J. Szekeley, A. W. Neumann, and Y. K. Chuang, "The rate of capillary penetration and the applicability of the Washburn equation," J. Colloid Interface Sci. 35, 273 (1971).

${ }^{14}$ G. I. Taylor, "An experimental study of standing waves," Proc. R. Soc. London 218, 44 (1953).

${ }^{15}$ B. W. Zeff, B. Kleber, J. Fineberg, and D. P. Lathrop, "Singularity dynamics in curvature collapse and jet eruption on a fluid surface," Nature (London) 403, 401 (2000). 